\title{
Review Article \\ Efficacy and Safety of Tripterygium Wilfordii Hook F on Psoriasis Vulgaris: A Systematic Review and Meta-Analysis of Randomized Controlled Trials
}

\author{
Meng Lv, ${ }^{1}$ Jingwen Deng, ${ }^{1,2}$ Nan Tang, ${ }^{1,3}$ Yuejin Zeng, ${ }^{1}$ and Chuanjian Lu $\mathbb{D}^{1,2,4}$ \\ ${ }^{1}$ Department of Dermatology, Guangdong Provincial Hospital of Chinese Medicine, Guangzhou 510120, China \\ ${ }^{2}$ Psoriasis Clinical and Basic Research Team, Guangdong Provincial Academy of Chinese Medical Sciences, Guangzhou 510120, China \\ ${ }^{3}$ Department of Dermatology, Guangzhou Red Cross Hospital, Guangzhou 510000, China \\ ${ }^{4}$ Guangdong Provincial Key Laboratory of Clinical Research on Traditional Chinese Medicine Syndrome, Guangzhou 510120, China
}

Correspondence should be addressed to Chuanjian Lu; luchuanjian888@vip.sina.com

Received 11 November 2017; Accepted 28 February 2018; Published 22 April 2018

Academic Editor: Deborah A. Kennedy

Copyright (c) 2018 Meng Lv et al. This is an open access article distributed under the Creative Commons Attribution License, which permits unrestricted use, distribution, and reproduction in any medium, provided the original work is properly cited.

\begin{abstract}
Background. Psoriasis is a chronic autoimmune-mediated skin disease that is characterized by persistent localized erythematous scaly plaque. Tripterygium wilfordii Hook F (TwHF), a well-known Chinese medicine that has been used for centuries in China to treat immune diseases, inflammation, and tumor, is accompanied by a degree of toxic effects. Its clinical efficacy and safety on psoriasis are incompletely understood. Aim. To summarize evidence concerning the efficacy and safety of TwHF in treating psoriasis. Methods. EMBASE, Ovid MEDLINE, PubMed, Web of Science, Springer, Cochrane Library, CNKI, CBM, Wanfang, and VIP database were searched up to October 2017. The included literature was assessed and extracted by two independent reviewers. To enhance the available evidence, a systematic review was performed to examine all relevant published literature relating to randomized controlled trials (RCTs) of TwHF. Relative ratios (RRs) and 95\% confidence intervals (CIs) were calculated, and a meta-analysis was conducted with RevMan 5.3 software. Results. Twenty eligible RCTs with 1872 participants were included for systematic review and meta-analysis. Studies were assessed using the Cochrane risk of bias tool. The meta-analysis of add-on effect of TwHF conferred benefit for psoriasis: combination treatment with compound glycyrrhizin (four studies, OR $=0.34,95 \% \mathrm{CI}$ $0.22-0.52, P<0.00001, I^{2}=0 \%$ ), combination treatment with acitretin (three studies, $\mathrm{OR}=0.25,95 \%$ CI $0.10-0.63, P=0.003$, $I^{2}=50 \%$ ), and combination treatment with compound amino-polypeptide tablet (three studies, OR $=0.37,95 \% \mathrm{CI} 0.22-0.63$, $\left.P=0.0002, I^{2}=0 \%\right)$. Conclusions. Despite several mild side effects of TwHF, there is evidence that TwHF is an effective therapy for psoriasis. However, the conclusions are limited by the small number of included trials. More well-designed RCTs with extensive follow-up periods are warranted to clarify the effects and safety of TwHF in treating psoriasis. Trial Registration. This review was registered in the International Prospective Register of Systematic Reviews (CRD42016041363).
\end{abstract}

\section{Introduction}

Psoriasis vulgaris is an immune-abnormal chronic skin disease characterized by well-delineated red, scaly plaques. Psoriasis affects approximately $2 \%-3 \%$ of the population worldwide, and it can significantly impair a participant's wellbeing and physical and mental functioning [1]. Convention therapies include nonspecific immunosuppressants, such as topical steroids and cyclophosphamide, and more specific compounds, such as cyclosporine or other drugs thought to act as immunomodulators (fumarates and intravenous immunoglobulins). There is increasing evidence for immunomodulatory and partly immunosuppressive mechanism of action of tripterygium wilfordii Hook F (TwHF), which has been widely used in China to treat various autoimmune and inflammatory diseases, including psoriasis, rheumatoid arthritis (RA), systemic lupus erythematosus (SLE), ankylosing spondylitis, and idiopathic IgA nephropathy [2]. Thus, compounds such as triptolide or triptolide derivatives may have the potential to be developed as a new class of drugs for immune diseases [3]. Currently, in clinical settings, the preparations of tripterygium wilfordii Radix are 
in the form of tripterygium, tripterygium total terpenoids, tripterygium glycosides, and tripterygium bilayer tablets, as well as triptolide ointment, which has great application value [2]. Tripterygium glycosides can be extracted as full root extract tripterygium tablets. Tripterygium tablets are used more to treat rheumatoid arthritis in the early stage, and tripterygium glycosides have the widest application. At present, tripterygium preparations are commonly used to treat various kidney disease, thyroid disease, rheumatism, and connective tissue disease.

In recent years, this medicine also has demonstrated applications in dermatology. The treatment of psoriasis with TwHF is well-accepted by clinicians; however, it lacks scientifically reliable evidence. This study provides a systematic review of all relevant published literature relating to randomized controlled trials (RCTs) of TwHF to further clarify the effect and safety of the TwHF treatment in psoriasis.

\section{Methods}

This review included all RCTs using TwHF on treatments of psoriasis. We compared TwHF either alone or in combination against a placebo or another active intervention. The primary outcome of this meta-analysis was the remission of psoriasis lesion.

2.1. Literature Search and Selection. A systematic search of articles was conducted using the following electronic databases: EMBASE, Ovid MEDLINE, PubMed, Web of Science, Springer, Cochrane Library, CNKI, CBM-Database, Wanfang database, and VIP database. Reference lists and citations of the identified studies were also screened for relevant publications and to investigate the efficacy and safety of TwHF in treating psoriasis.

2.2. Eligibility Criteria. Studies were included if they were prospective RCTs that compared orally administered TwHF (either alone or in combination with other active interventions) with placebo or active medicine.

2.3. Exclusion Criteria. Studies were excluded if they met the following exclusion criteria:

(1) Participants had comorbidities.

(2) The publications lacked original data for the metaanalysis and review articles.

(3) The data did not predominantly concern vulgaris type psoriasis (e.g., Guttate, pustular, erythrodermic, inverse, and nail psoriasis).

(4) TwHF was used externally.

2.4. Study Selection. In the first stage of the study selection process, the titles and abstracts of the papers searched by keywords were examined to exclude irrelevant articles. Next, the full texts of all selected studies were screened according to the inclusion and exclusion criteria.

2.5. Data Extraction and Quality Assessment. Using a data extraction form developed in advance, two reviewers (Lv and
Deng) independently extracted the following information: first author, publication year, sample size, participant age, intervention, duration, and outcome. The main outcome was the reduction of Psoriasis Area and Severity Index (PASI) (Table 1). Adverse events were also recorded (Table 2). Two reviewers (Lv and Deng) independently conducted risk of bias assessments using the Cochrane Collaboration tool for assessing risk of bias.

2.6. Statistical Analysis. Meta-analysis was performed in RevMan (Version 5.3. Copenhagen: The Nordic Cochrane Centre, The Cochrane Collaboration, 2014). Risk ratios (RR) with $95 \%$ confidence intervals (CI) for dichotomous data and mean differences (MD) with 95\% CIs for continuous data were reported. For studies on a similar type of intervention, we conducted meta-analysis using a fixed-effect model to calculate a pooled intervention effect estimate across trials when the $I^{2}$ statistic was less than $50 \%$ with reasonable clinical homogeneity. If the $I^{2}$ statistic was $50 \%$ to $80 \%$, we applied a random-effects model [4]. Meta-analysis was performed according to the different interventions, such as TwHF alone or in combination. And different combinations (compound glycyrrhizin (CG), acitretin, and compound amino-polypeptide tablet (CAPT)) were considered as different interventions.

\section{Results}

3.1. Literature Search. After removing duplicates, we identified 1845 references from our electronic searches (Figure 1). 1683 articles remained after duplicates were removed. After scanning titles and abstracts, we discarded 1530 articles because of irrelevant topics or noncomparative studies. Next, by full-text articles assessing, 136 articles were excluded because they were not for psoriasis vulgaris; did not include appropriate comparator; did not report specified outcomes; or are without useable data. Among the remaining 20 publications for the systematic review, only 11 publications included quantitative synthesis meta-analyses.

3.2. Description of Studies. The 20 included RCTs, involving 1872 participants with psoriasis vulgaris, were conducted in hospitals in China and published in Chinese from 1999 to October 2017. Characteristics of the included studies are summarized in Table 1. One study [5] was a randomized, doubleblind, double-dummy, parallel-group clinical trial. And only this mentioned the details of random sequence generation and allocation concealment. Three studies [5-7] designed the blinding of participants and outcome assessment. The sample sizes for the included studies ranged from 39 to 200, and the participant age interval ranged from 16 to 78 years.

3.3. Description of Interventions. Among the included studies, placebo was used as control only in one study [7]. Four studies $[5,6,8,9]$ reported TwHF as monotherapy compared active interventions (cyclosporin A, acitretin, CG, and Qingdai capsule). There were five studies [10-14] that included the comparison of TwHF plus CG and CG. Three studies were compared TwHF alone or plus acitretin with 


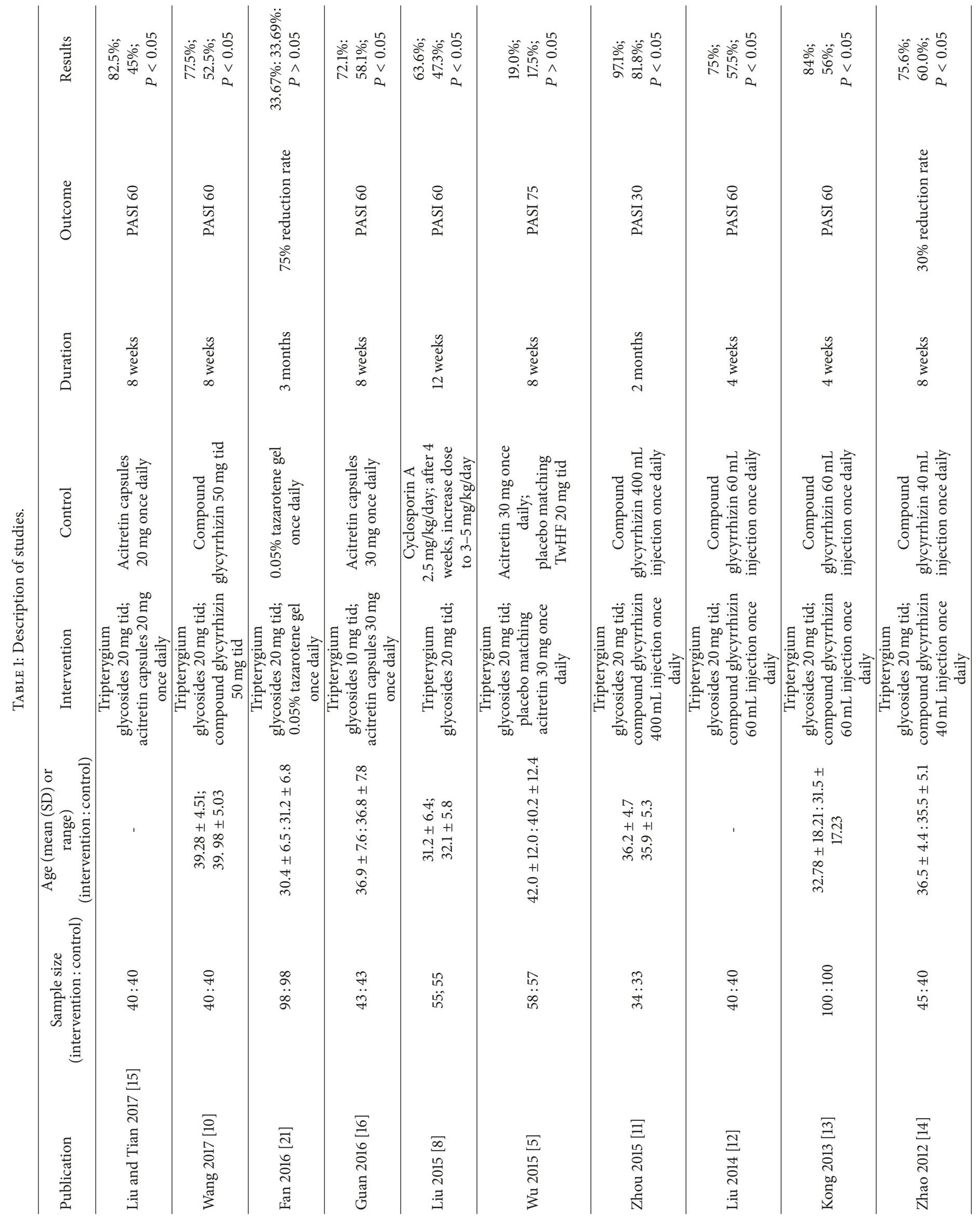




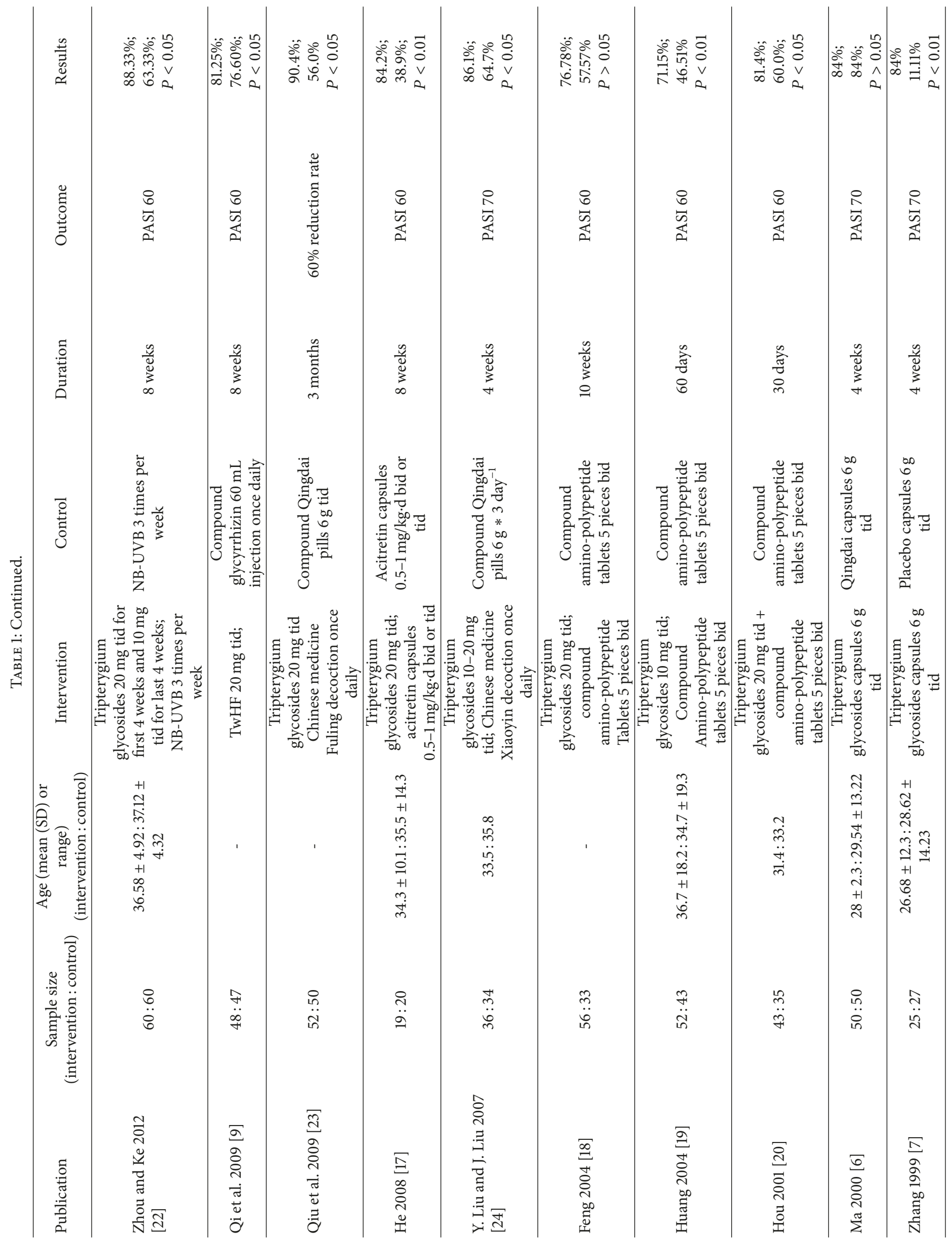




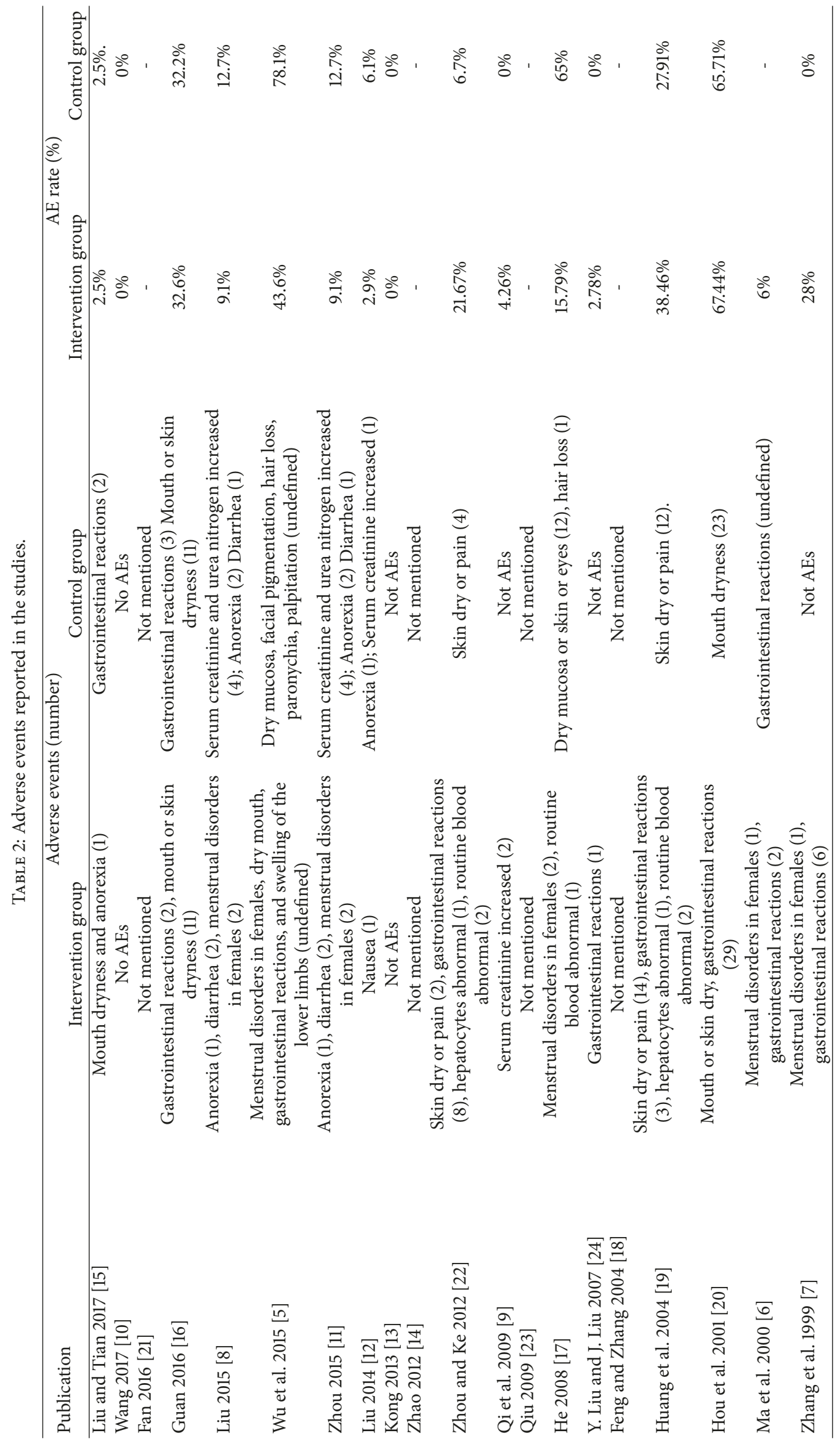




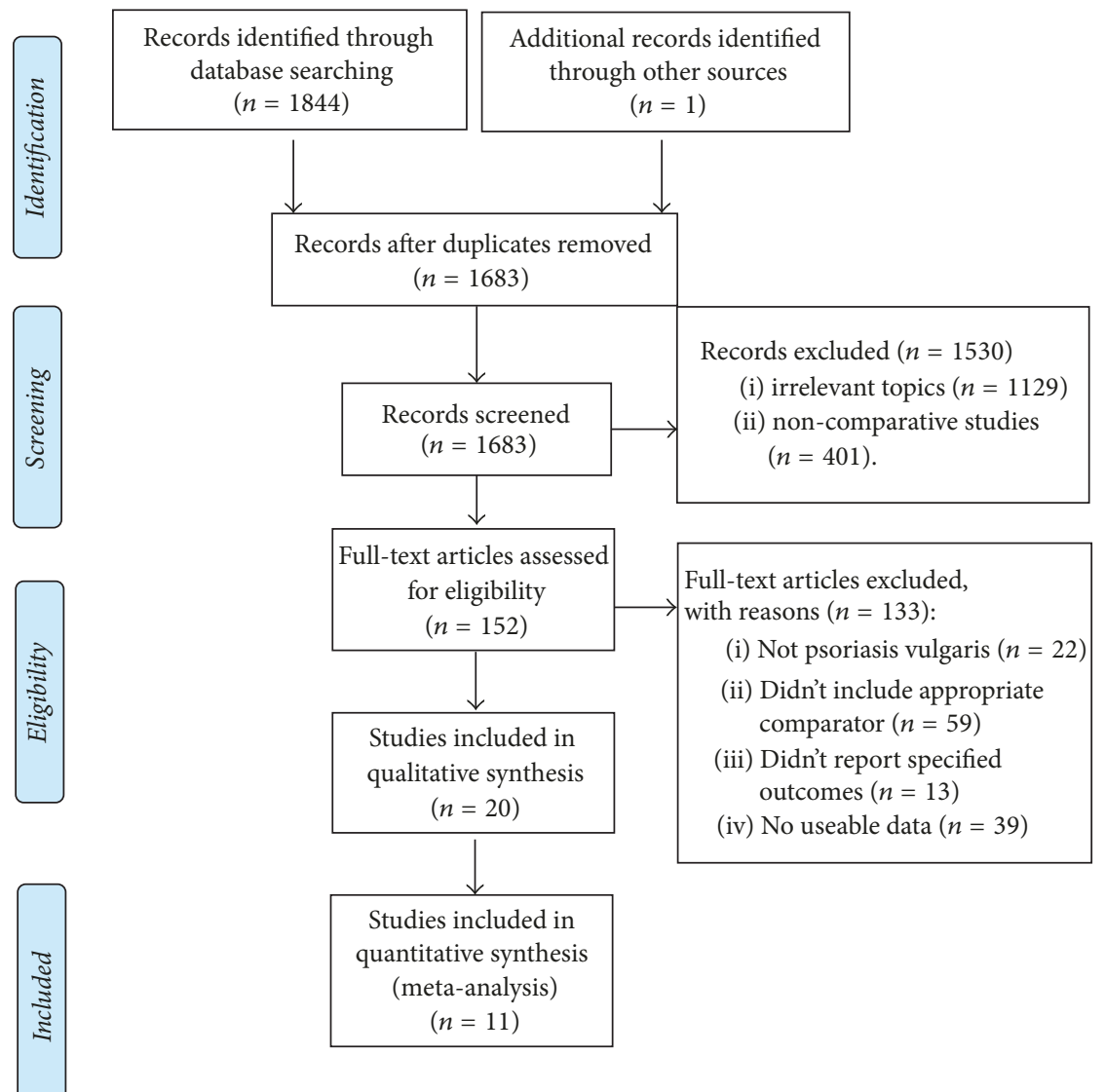

FIGURE 1: A PRISMA flowchart of the study selection process.

acitretin [15-17]. In three studies [18-20], TwHF plus CAPT was compared with CAPT alone. Other studies [21-24] combined TwHF with conventional therapies: tazarotene, NB-UVB, and traditional Chinese medicine.

3.4. Outcome Measurements. The reduction of disease severity was assessed based on the change in the lesion reduction.

PASI score was used in clinical trials to measure outcomes following psoriasis treatment. The PASI measures the redness, thickness, and scaling of the lesions and the area of involvement, with a total score ranging from 0 to 72. PASI-60, referring to a $60 \%$ reduction in PASI, was considered to be effective in treating psoriasis treatment. Twelve of the twenty studies [8-10, 12, 13, 15-20, 22] reported PASI-60 as primary outcome, which was consistent with the Consensus of Diagnosis and Treatment of Psoriasis Vulgaris with Integrative Medicine [25]. One study [5] reported PASI75 and three studies $[6,7,24]$ reported PASI-70 as primary outcome.

According to the disease of TCM syndrome diagnosis curative standard, skin lesions that reduced $60 \%$ or above were also accepted as being effective. Other three studies $[14,21,23]$ used skin lesions reduction as the effectiveness indicators.

Of all studies, two studies reported the relapse rates at the end of the follow-up period $[6,24]$, but the definitions of relapse were not stated.
3.5. Dropouts and Withdrawals. Wu et al. 2015 [5] reported that there was a higher attrition rate in the acitretin group (5.2\% in the TwHF group and $7.0 \%$ in the acitretin group by week 2 , and $8.6 \%$ in the TwHF group and $12.3 \%$ in the acitretin group by week 8 . Discontinuation occurred because of withdrawn consent $(n=1)$, loss to follow-up $(n=8)$, adverse event (AE) $(n=1)$, and disease progression $(n=2)$. The reasons for discontinuing the study were similar between two groups. He 2008 [17] reported two withdrawals because of AEs in the acitretin group, one because of high cholesterol and one because of serious cheilitis. Zhang et al. 1999 [7] reported that two people withdrew in the TwHF group and that they had gastrointestinal-related adverse reactions. No dropouts were reported in the other studies.

3.6. Risk of Bias Assessment. Only two studies [5, 15] stated the details of random sequence generation and one study [5] clarified allocation concealment, and others remained unclear. Attempts were made to contact the original authors to obtain further information about randomization and allocation on the unclear studies. However, the authors of the studies could not be reached.

For "sequence generation," two studies $[5,15]$ stated that they used random number tables generated by computer which were assessed as "low risk"; the others were assessed as "unclear." And beside Wu et al. 2015, the allocation concealment of other studies was not clearly defined. Therefore, 
the other nineteen studies were assessed as "unclear" in "allocation concealment" because of the lack of information. Three studies that used double-dummy methods or placebo [5-7] were assessed as "low risk" in "blinding," and the other studies were assessed as "high risk" because of different types of dosage forms or combinations with other types of medicines. Three study $[5,7,17]$ had withdrawals in the trial, and they were assessed as "high risk" for "incomplete outcome data." And the other studies were assessed as "unclear." All studies were assessed as "low risk" for "selective reporting" because they reported the outcomes prespecified in the methods. All studies were assessed as "low risk" for "other bias," which refers to the inappropriate influence of cofounders.

Risk of bias assessments is presented in Figure 2.

\subsection{Effects of Interventions}

3.7.1. TwHF Alone for Psoriasis Vulgaris. Only one study [19] compared TwHF with placebo. Results of the study showed that there was significant clinical improvement in participants treated with TwHF. No severe side effects of TwHF were observed. Four studies $[5,6,8,9]$ compared TwHF with other active interventions. Liu 2015 [8] showed that tripterygium glycosides could improve psoriasis by inhibiting the activation of $\mathrm{T}$ lymphocytes, and its effect and safety were better than cyclosporine A. Wu et al. 2015 [5] conducted a randomized, double-blind, double-dummy, parallel-group clinical trial to prove that there was no significant difference in treatment efficacy between the TwHF and acitretin, but there were fewer treatment-related adverse events in the TwHF group. In Qi et al. 2009 [9], it was equivalence effect of TwHF and glycyrrhizin. In Ma et al. 2000 [6], comparing with Qingdai capsules, tripterygium wilfordii had a fast effect on advanced psoriasis, but long-term follow-up was needed for its curative effect.

\subsubsection{Add-On Effect on TwHF for Psoriasis Vulgaris}

(1) TwHF plus CG for Psoriasis Vulgaris. The main component of CG, glycyrrhizic acid, a triterpenoid saponin glycoside, consists of one molecule of $18 \beta$-glycyrrhetic acid as aglycon and two molecules of glucuronic acid, which is known as the most efficacious composition of licorice [26, 27]. Glycyrrhizic acid has been proved to have antiallergic, antiviral, and antiinflammatory activities $[28,29]$. There were four studies [10-14] which included the comparison of TwHF plus CG and CG alone. The results showed that the participants in combination therapy group got more benefit than in the GC group (Figure 3, four studies, $\mathrm{OR}=0.34,95 \%$ CI $0.22-0.52$, $P<0.00001, I^{2}=0 \% ; P=0.02, I^{2}=11 \% ; P<0.00001$, $\left.I^{2}=0 \%\right)$.

(2) TwHF plus Acitretin for Psoriasis Vulgaris. Acitretin is the pharmacologically active metabolite of etretinate, which is highly efficacious as monotherapy in psoriasis and currently approved by the FDA. It has dose-sparing effects when used as combination therapy with conventional systemic drugs. It is a good option for long-term maintenance therapy [30]. When TwHF plus acitretin was compared with acitretin alone
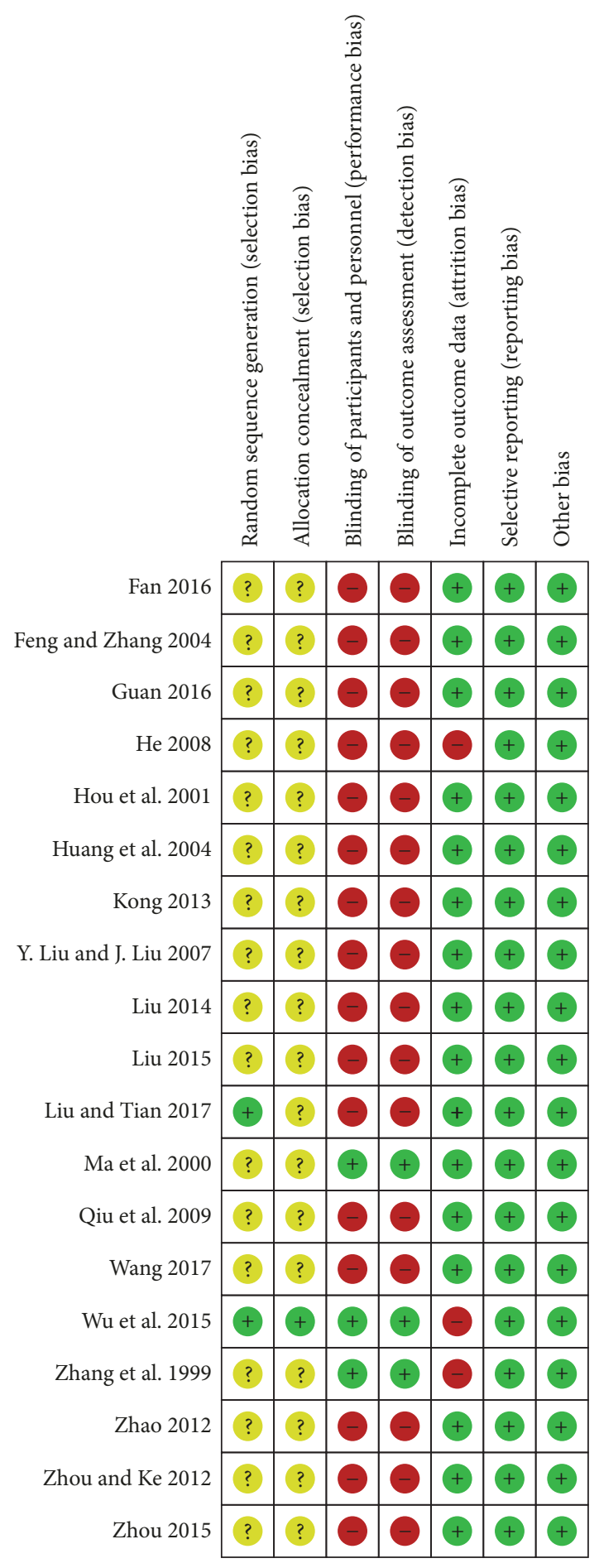

FIGURE 2: Risk of bias summary.

in three studies [15-17], the pooled result of PASI-60 was statistically significant (Figure 4, three studies, $\mathrm{OR}=0.25$, $95 \%$ CI $\left.0.10-0.63, P=0.003, I^{2}=50 \%\right)$.

(3) TwHF plus CAPT for Psoriasis Vulgaris. The main component of CAPT, aminopeptidase, is an active substance extracted from animal organs. It contains a variety of amino acids, peptides, and trace elements, which helps to regulate 


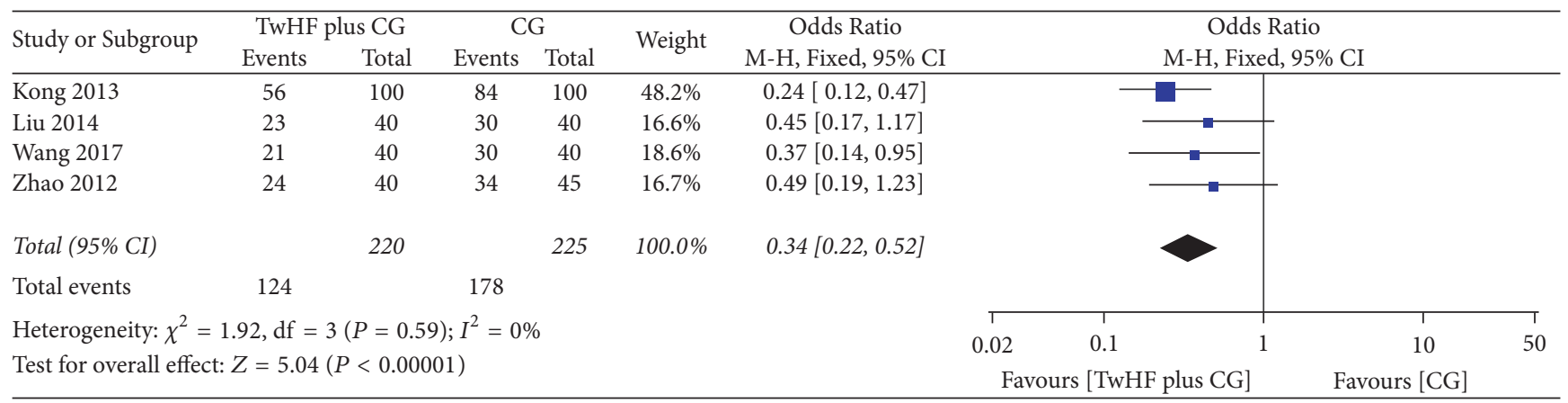

FIGURE 3: Effect of TwHF plus CG for psoriasis vulgaris.

\begin{tabular}{|c|c|c|c|c|c|c|c|c|c|c|}
\hline \multirow{2}{*}{ Study or Subgroup } & \multicolumn{2}{|c|}{ Experimental } & \multicolumn{2}{|c|}{ Control } & \multirow{2}{*}{ Weight } & \multirow{2}{*}{$\begin{array}{c}\text { Odds Ratio } \\
\text { M-H, Random, 95\% CI }\end{array}$} & \multirow{2}{*}{\multicolumn{4}{|c|}{$\begin{array}{c}\text { Odds Ratio } \\
\text { M-H, Random, 95\% CI }\end{array}$}} \\
\hline & Events & Total & Events & Total & & & & & & \\
\hline Guan 2016 & 25 & 43 & 31 & 43 & $40.7 \%$ & & \multicolumn{4}{|c|}{\begin{tabular}{l|l}
$\square$ &
\end{tabular}} \\
\hline He 2008 & 7 & 18 & 16 & 19 & $22.9 \%$ & \multicolumn{2}{|l|}{$0.12[0.03,0.57]$} & 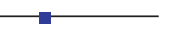 & & \\
\hline Liu and Tian 2017 & 18 & 40 & 33 & 40 & $36.4 \%$ & \multicolumn{2}{|l|}{$0.17[0.06,0.48]$} & & & \\
\hline \multicolumn{2}{|l|}{ Total (95\% CI) } & 101 & & 102 & $100.0 \%$ & \multirow[t]{2}{*}{$0.25[0.10,0.63]$} & & & & \\
\hline Total events & 50 & & 80 & & & & & & & \\
\hline \multirow{2}{*}{\multicolumn{7}{|c|}{$\begin{array}{l}\text { Heterogeneity: } \tau^{2}=0.33 ; \chi^{2}=4.01, \mathrm{df}=2(P=0.13) ; I^{2}=50 \% \\
\text { Test for overall effect: } Z=2.94(P=0.003)\end{array}$}} & 0.01 & 0.1 & 10 & 100 \\
\hline & & & & & & & Fav & HF plus Acitreti & Favours $[\mathrm{Ac}$ & \\
\hline
\end{tabular}

FIGURE 4: Effect of TwHF plus acitretin for psoriasis vulgaris.

the immune function of the body and is conducive to the body's nutritional metabolism. In three studies [18-20], TwHF plus CAPT was compared with CAPT alone. The pooled result of PASI-60 was statistically significant (Figure 5, three studies, $\mathrm{OR}=0.37,95 \%$ CI $0.22-0.63, P=0.0002$, $\left.I^{2}=0 \%\right)$.

3.8. Adverse Events. Fourteen studies reported AEs (Table 2). Four studies did not mention any information about AE $[14,18,21,23]$. Two studies reported that there were no AEs in the two groups $[10,13]$. In the TwHF group, the frequent complaints were menstrual disorders in females, dry mouth, gastrointestinal complaints, swelling of the lower limbs, abnormal hepatocytes, and abnormal routine blood results; several studies clearly reported the numbers of different AEs, while some only recorded the AEs and did not provide accurate numbers $[5,6]$. Two participants in the control group (acitretin group) withdrew because of AEs, one had high cholesterol, and one had serious cheilitis [17]. Another study reported that one participant in the acitretin group withdrew because of AEs [5]. Two participants withdrew in the TwHF group, both of them because of gastrointestinal adverse reactions [7]. Most of the AEs returned to normal without additional treatment by reducing or stopping the dosage of intervention. However, the relationships between the AEs and the interventions were not further discussed in any study. No severe AEs were reported.

\section{Discussion}

Psoriasis vulgaris is a chronic immune-mediated cutaneous inflammatory disease. TwHF is often used to treat a variety of immune and inflammation-related diseases, such as psoriasis and RA in China due to their favorable cost benefit ratio. The extracts of TwHF contain more than 70 ingredients; however, triptolide is the most potent bioactive substance [31, 32]. It has been shown to possess potent anti-inflammatory and immunosuppressive properties in vitro, as well as in different animal models in numerous preclinical studies [33]. Numerous preclinical studies have demonstrated that the extracts from the root of TwHF inhibit the expression of proinflammatory cytokines, proinflammatory mediators, adhesion molecules, and matrix metalloproteinases by macrophages, lymphocytes, synovial fibroblasts, and chondrocytes. TwHF also induces apoptosis in lymphocytes and synovial fibroblasts and inhibits their proliferation [2]. The major side effects of TwHF reported in the literature are skin dry and rash, gastrointestinal complaints, decreases in erythrocytes and leukocytes, and reproductive toxicity such as dysmenorrhea, irregular menstruation, or reversible sterility. However, serious adverse events have rarely been reported [33].

From the meta-analysis, tripterygium wilfordii Hook $\mathrm{F}$ might be an effective and safe treatment in participants with psoriasis vulgaris. In this review, we focused on the efficacy and safety of TwHF. Five studies [5-9] reported TwHF as monotherapy compared placebo or active interventions. Some medicines (cyclosporin A, acitretin, CG, and Qingdai capsule) used as control were regarded as an effective drug treatment for psoriasis. We did not pool data for these five studies because the medicines used in control group were not comparable. However, these studies indicated TwHF was effective in psoriasis vulgaris. Differing from the results of TwHF used as monotherapy, when TwHF combined with 


\begin{tabular}{|c|c|c|c|c|c|c|c|c|c|c|}
\hline \multirow{2}{*}{ Study or Subgroup } & \multicolumn{2}{|c|}{ TwHF plus CAPT } & \multicolumn{2}{|c|}{ CAPT } & \multirow{2}{*}{ Weight } & \multirow{2}{*}{$\begin{array}{c}\text { Odds Ratio } \\
\text { M-H, Fixed, 95\% CI }\end{array}$} & \multirow{2}{*}{\multicolumn{4}{|c|}{$\begin{array}{c}\text { Odds Ratio } \\
\text { M-H, Fixed, 95\% CI }\end{array}$}} \\
\hline & \multirow{2}{*}{$\frac{\text { Events }}{19}$} & \multirow{2}{*}{$\begin{array}{c}\text { Total } \\
33\end{array}$} & \multirow{2}{*}{$\frac{\text { Events }}{43}$} & \multirow{2}{*}{$\frac{\text { Total }}{56}$} & & & & & & \\
\hline Feng and Zhang 2004 & & & & & $30.7 \%$ & $0.41[0.16,1.04]$ & & 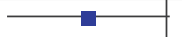 & & \\
\hline Hou et al. 2001 & 21 & 35 & 35 & 43 & $28.5 \%$ & $0.34[0.12,0.95]$ & & & & \\
\hline Huang et al. 2004 & 20 & 43 & 37 & 52 & $40.7 \%$ & $0.35[0.15,0.82]$ & & & & \\
\hline Total (95\% CI) & & 111 & & 151 & $100.0 \%$ & $0.37[0.22,0.63]$ & & & & \\
\hline Total events & 60 & & 115 & & & & & & & \\
\hline Heterogeneity: $\chi^{2}=0$. & $08, \mathrm{df}=$ & $P=0.96$ & $I^{2}=0 \%$ & & & & 0.05 & 0.2 & $\begin{array}{l}1 \\
5\end{array}$ & 20 \\
\hline Test for overall effect: 2 & $=3.68$ & $=0.000$ & & & & & Favour & HF plus CAPT] & Favours & \\
\hline
\end{tabular}

FIgURE 5: Effect of TwHF plus CAPT for psoriasis vulgaris.

other medicines, such as CG, acitretin, and CAPT, the quantitatively assessment showed that the effect of intervention groups was better than that of the control groups.

More than one in seventh patients who were taking TwHF had experienced AEs. Menstrual disorders in females, dry mouth, and gastrointestinal complaints were the most commonly reported AEs following oral TwHF; 14 people had menstrual disorders in 7 studies, but none of them withdrew from the studies. Compared with acitretin, TwHF treatment might have a lower incidence of AE. Gastrointestinal reactions were reported in the two groups. Two participants in TwHF group withdrew from the studies because of serious gastrointestinal reactions, but both of them did not need other treatment. Some participants were unnormal in physical examination such as liver function and routine blood test. All participants returned to normal after reducing the dosage of the medicine or stopping it completely. TwHF should not be used for prolonged periods of time because it will increase risk of adverse reproductive outcomes and its enteron irritation.

Although TwHF was effective in treating psoriasis, the design of many of the studies, which have been published and discussed, may not meet the state-of-the-art criteria for clinical trials today. In addition, significant improvement of PASI in participants with only mild forms of psoriasis may bias a favorable efficacy outcome. Another drawback in better understanding the benefit-risk profile of triptolide is the lack of long-term trials or even reports of open studies lasting for more than one year. Based on published data to date, it is difficult to judge the full potential of this drug for use in psoriasis. Most of the studies did not clearly mention "the sequence generation" and "blinding"; therefore, most of them were low quality studies. Moreover, the sample sizes of the included studies were not sufficiently large.

\section{Conclusions}

This meta-analysis indicated that tripterygium wilfordii Hook F might be an effective and safe treatment in participants with psoriasis. Nevertheless, despite our rigorous methodology, the inherent limitations of the included studies prevent us from reaching a definitive conclusion. Future large-volume, well-designed RCTs with extensive follow-up periods are warranted to confirm and update the findings of this analysis.

\section{Disclosure}

Meng Lv and Jingwen Deng are co-first authors.

\section{Conflicts of Interest}

There are no conflicts of interest.

\section{Acknowledgments}

This study is supported by the National Natural Science Foundation of China (no. 81603619), Chinese Medicine Scientific Research Project of Guangdong Province (no. 20161096), and Special Funding for TCM Science and Technology Research of Guangdong Provincial Hospital of Chinese Medicine (nos. YN2015QN01 and YN2016QJ02).

\section{References}

[1] M. Enamandram and A. B. Kimball, "Psoriasis epidemiology: The interplay of genes and the environment," Journal of Investigative Dermatology, vol. 133, no. 2, pp. 287-289, 2013.

[2] J. Bao and S. M. Dai, "A Chinese herb Tripterygium wilfordii Hook $\mathrm{F}$ in the treatment of rheumatoid arthritis: mechanism, efficacy, and safety," Rheumatology International, vol. 31, no. 9, pp. 1123-1129, 2011.

[3] M. Weiguang, Z. Tao, Z. Chao et al., "Research and Prospect on Poisonous Herb Tripterygium Wilfordii," China Journal of TCM and Pharmacy, vol. 21, no. 2, pp. 117-120, 2006.

[4] J. P. T. Higgins and S. G. Thompson, "Quantifying heterogeneity in a meta-analysis," Statistics in Medicine, vol. 21, no. 11, pp. 15391558, 2002.

[5] C. Wu, H. Jin, D. Shu et al., "Efficacy and safety of Tripterygium wilfordii Hook F versus acitretin in moderate to severe psoriasis vulgaris: A randomized clinical trial," Chinese Medical Journal, vol. 128, no. 4, pp. 443-449, 2015.

[6] Y. Ma, S. Cheng, and X. Wang, "A double-blind research on the therapeutic effect of Tripterygium wilfordii HookF on progressive stage psoriasis vulgaris," Chinese Journal of Convalescent Medicine, vol. 9, pp. 33-35, 2000.

[7] F. Zhang, R. Tian, S. Ma et al., "Treatment of active psoriasis vulgaris with Tripterygium wilfordii: double blind controlled trial," American Journal of Clinical Dermatology, vol. 28, pp. 3233, 1999.

[8] W. Liu, "Effect of tripterygium glycosides treatment of psoriasis vulgaris and its influence on T cell subsets," Modern Journal of 
Integrated Traditional Chinese and Western Medicine, vol. 24, no. 3, pp. 318-320, 2015.

[9] Y. Qi, H. Wang, S. Hou, and Q. Liu, "Efficacy of stronger neo-minophagen $\mathrm{c}$ in treatment of psoriasis vulgaris," Chinese Journal of Dermatovenereology, vol. 23, no. 2, pp. 1-2, 2009.

[10] H. Wang, "Clinical efficacy of tripterygium glycosides combined with Compound Glycyrrhizin in the treatment of psoriasis vulgaris," Journal of Medical Research and Practice, vol. 30, no. 8, pp. 1178-80, 2017.

[11] Y. Zhou, "Clinical observation of Compound Glycyrrhizin Combined with Tripterygium Glycosides in the treatment of psoriasis vulgaris," China Medical Engineering, vol. 23, no. 4, pp. 121-123, 2015.

[12] S. Liu, "Clinical observation of Compound Glycyrrhizin Combined with Tripterygium wilfordii tablet in the treatment of psoriasis vulgaris," Chinese Journal of Modern Drug Application, vol. 8, no. 1, pp. 138-139, 2014.

[13] L. Kong, "Clinical Observation of Glycyrrhizin Monoammonium Combined with Tripterygium in Treatment of Psoriasis," Clinical Rational Drug Use, vol. 6, no. 2C, pp. 4-5, 2013.

[14] J. Zhao, "Clinical observation of compound glycyrrhizin combined with tripterygium glycosides in the treatment of psoriasis vulgaris," China Foreign Medical, vol. 12, p. 123, 2012.

[15] J. Liu and H. Tian, "The clinical effect of tripterygium glycosides combined with acitretin A in the treatment of psoriasis vulgaris and its effect on immune cytokines," Journal of Chifeng University, vol. 33, no. 2, pp. 72-73, 2017.

[16] X. Guan, "The Effect of Acitretin Combined With Tripterygium Glycosides on Psoriasis," China Health Standard Management, vol. 7, no. 22, pp. 131-133, 2016.

[17] J. He, "Comparison of Tripterygium glycoside and acitretin effect in treatment of psoriasis vulgaris," Journal of Pharmacy Practice and Community Medicine, vol. 25, no. 10, pp. 1188-1189, 2008 (Chinese).

[18] P. Feng and Y. Zhang, "Observation of therapeutic effect of treating psoriasis with compound amino-polypeptide tablet Plus Tripterygium wilfordii hook," Journal of Community Medicine, vol. 2, no. 1, p. 85, 2004.

[19] Y. Huang, F. Huang, and D. Ma, "Clinical observation of 52 cases of psoriasis vulgaris treated with compound amino-polypeptide tablet combined with Tripterygium wilfordii Hook," Chinese Journal of traditional Chinese and Western Medicine, vol. 2, no. 3, pp. 238-239, 2004.

[20] J. Hou, D. Zhang, and S. Wu, "Effect of combined treatment of psoriasis vulgaris with compound amino-polypeptide tablet and Tripterygium wilfordii hook," Medical Journal of Qilu, vol. 16 , no. 4, p. 327, 2001.

[21] Q. Fan, "Analysis of curative effect by tripterygium wilfordii glycosides tablet combined with tazarotene in thetreatment of psoriasis vulgaris," Chinese Journal of Modern Drug Application, vol. 10, no. 4, pp. 8-9, 2016.

[22] Z. Zhou and Q. Ke, "Clinical observation of NB-UVB combined with Tripterygium Wilfordii in the treatment of psoriasis vulgaris," Hainan Medical Journal, vol. 23, no. 2, pp. 82-83, 2012.

[23] G. Qiu, D. Wang, and L. Zhang, "Chinaroot soup coordination tripterygium wilfordii multi-glucoside treating psoriasis curative," Liaoning Journal of traditional Chinese Medicine, vol. 36, no. 3, pp. 394-395, 2009.

[24] Y. Liu and J. Liu, "Clinical observation on the treatment of psoriasis vulgaris with Xiao Yin decoction combined with the tripterygium glycoside tablet," China journal of Leprosy and Skin Diseases, vol. 23, no. 10, pp. 927-928, 2007.
[25] Dermatology Committee of People's Liberation Army Association of Traditional Chinese Medicine, "Consensus on diagnosis and treatment of chinese integrative medicine for psoriasis vulgaris," Chinese Journal of Dermatology, vol. 8, p. 328, 2009.

[26] W. T. Chung, S. H. Lee, J. D. Kim et al., "Effect of the extracts from Glycyrrhiza uralensis Fisch on the growth characteristics of human cell lines: Anti-tumor and immune activation activities," Cytotechnology, vol. 37, no. 1, pp. 55-64, 2001.

[27] S.-M. Ju, M.-S. Kim, Y.-S. Jo et al., "Licorice and its active compound glycyrrhizic acid ameliorates cisplatin-induced nephrotoxicity through inactivation of p53 by scavenging ROS and overexpression of p21 in human renal proximal tubular epithelial cells," European Review for Medical and Pharmacological Sciences, vol. 21, no. 4, pp. 890-899, 2017.

[28] J.-Y. Li, H.-Y. Cao, P. Liu, G.-H. Cheng, and M.-Y. Sun, "Glycyrrhizic acid in the treatment of liver diseases: literature review," BioMed Research International, vol. 2014, Article ID 872139, 15 pages, 2014.

[29] S. Li, J.-H. Zhu, L.-P. Cao et al., "Growth inhibitory in vitro effects of glycyrrhizic acid in U251 glioblastoma cell line," Neurological Sciences, vol. 35, no. 7, pp. 1115-1120, 2014.

[30] S. Subedi, Q. Yu, Z. Chen, and Y. Shi, "Management of pediatric psoriasis with acitretin: a review," Dermatologic Therapy, 2017.

[31] J. R. Zheng, J. L. Fang, K. X. Gu et al., "Screening of active anti-inflammatory-immunosuppressive and antifertile compositions from Tripterygium wilfordii. I. Screening of 8 components from total glucosides of Tripterygium wilfordii (TII)," Acta Academiae Medicinae Sinicae, vol. 9, no. 5, pp. 317322, 1987.

[32] Y. Y. Fan and S. Chen, "Immunological effects of Tripterygium wilfordii Hook F," Chinese Journal of Experimental and Clinical Immunology, vol. 2, p. 40, 1990.

[33] R. Han, M. Rostami-Yazdi, S. Gerdes, and U. Mrowietz, "Triptolide in the treatment of psoriasis and other immunemediated inflammatory diseases," British Journal of Clinical Pharmacology, vol. 74, no. 3, pp. 424-436, 2012. 


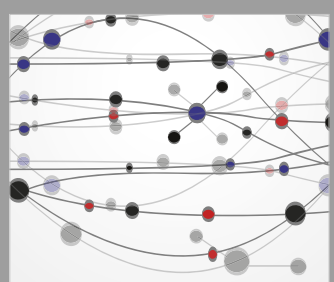

The Scientific World Journal
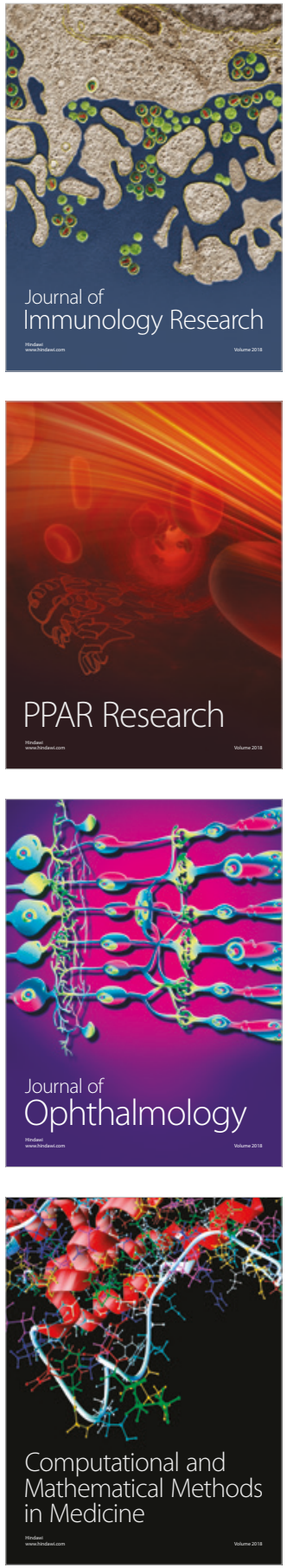

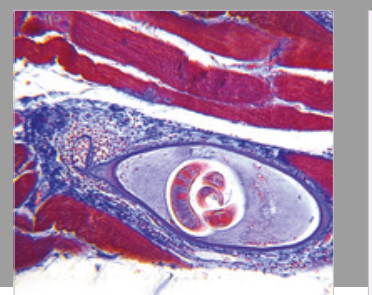

Gastroenterology Research and Practice

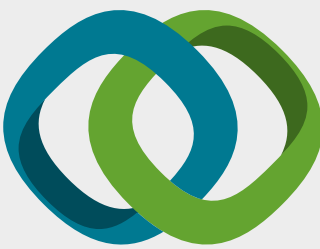

\section{Hindawi}

Submit your manuscripts at

www.hindawi.com
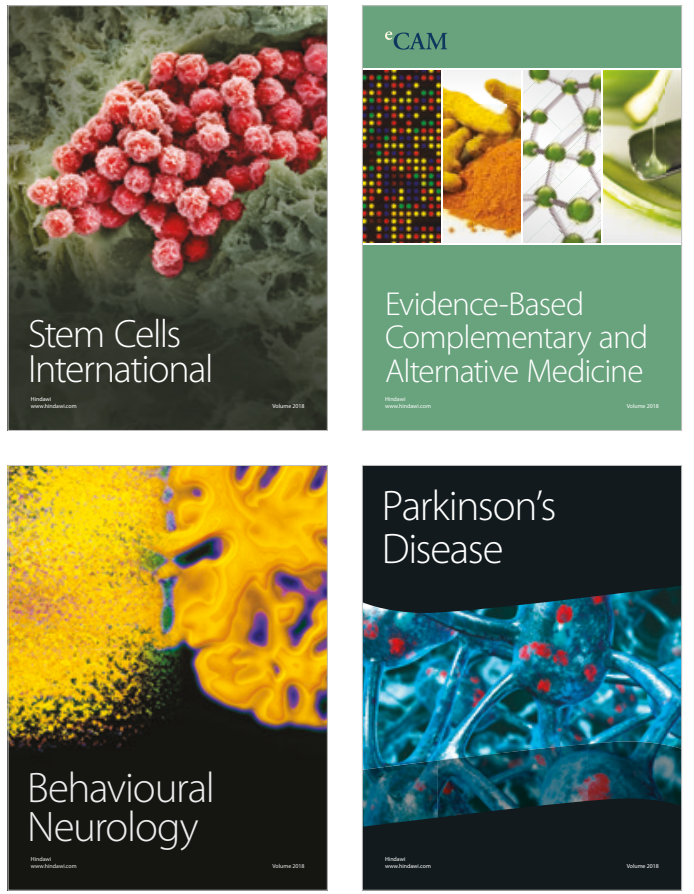

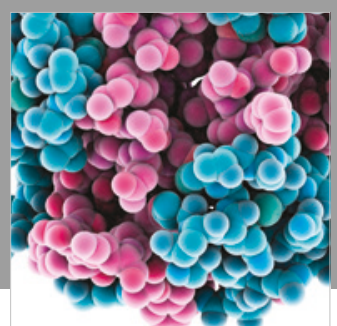

ournal of

Diabetes Research

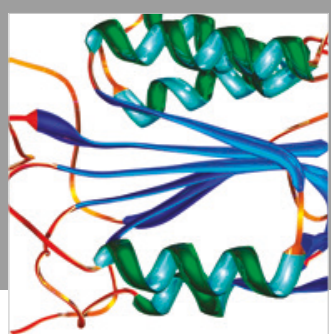

Disease Markers
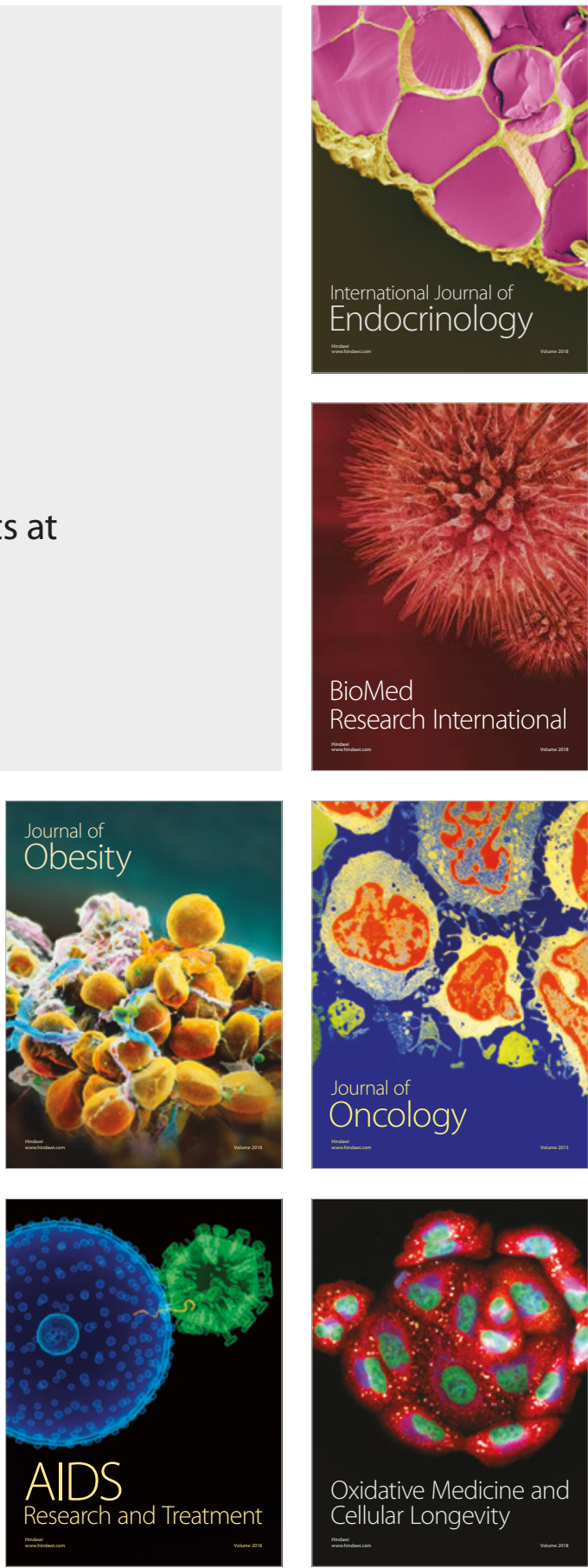